\title{
Relationship Between IL-2, IL-17 Concentrations, and Serum Creatinine Levels in Men with Chronic Kidney Disease
}

\author{
Khalid Farouq Al-rawi ${ }^{1}$, Hameed Hussein $\mathrm{Ali}^{1}$, \\ Manaf Abdulrahman Guma ${ }^{2}$, Bilal Jasir Mohammed Aldahham², \\ Shakir Faris Tuleab Alaaraji ${ }^{3}$, Osamah Al-ani ${ }^{4}$, Aus Tariq Ali*5
}

\begin{abstract}
Background: Chronic kidney disease (CKD), is a major public health challenge worldwide. It is more prevalent in developed countries compared with the rest of the world, due to the higher rates of life expectancy and unhealthy lifestyle related factors. This aim of the current study is to evaluate the relationship between interleukins IL-2 and IL-17 concentrations and kidney function markers in men with CKD.

Methods: Forty-five men with CKD and seventy controls were enrolled in the current study to assess the relationship between interleukin-2 (IL-2), interleukin-17 (IL-17), and CKD parameters. Fasting blood samples were collected from patients with CKD and their controls at same time. Serum IL-2, and IL-17 were measured in patients with CKD and their controls, and then the relationship between these interleukins and serum creatinine, serum urea, serum uric acid and urine albumin were evaluated.

Results: A significant relationship was detected between IL-2 ( $p<0.001)$, IL-17 ( $p<0.001)$ levels and serum creatinine concentrations. The significant increase of IL-2 and IL-17 levels were also paralleled with a significant increase in serum urea $(\mathrm{p}<0.001)$, and urine albumin $(\mathrm{p}<0.001)$ concentrations respectively.

Conclusions: IL-2 and IL-17 may play a critical role in the pathophysiology of CKD. The significant increase of IL-2 and IL-17 is associated with significantly high concentrations of creatinine, serum urea and urine albumin suggesting that these interleukins may be used as targets for future biomarkers and molecular therapy. However, due to limited sample size of the current study, larger prospective cohorts are needed to confirm these observations.
\end{abstract}

Keywords: Chronic kidney disease, Interleukins, Serum creatinine, Serum urea, Urine albumin.

\section{Introduction}

Chronic kidney disease (CKD), is a major public health problem, affecting 10 to $15 \%$ of the general population worldwide and its incidence rate is increasing $(1,2)$. Compared with the rest of the world, CKD is more prevalent in developed countries due to the higher rates of life expectancy and unhealthy lifestyle related factors $(3,4)$. Developed countries have high prevalence of lifestyle related diseases such as obesity, hypertension, type 2 diabetes (T2D) and cardiovascular disease (CVD), which are all known to be an established risk factors for $\operatorname{CKD}(5,6,7)$. The pathophysiology of CKD is very complex and is commonly influenced by exogenous and endogenous factors and their interaction. 
Cytokines are among the endogenous factors that are produced predominantly by macrophages $(8,9)$. Regardless of the initiation cause, CKD development is associated with a significantly higher level of inflammation leading to an activation of the immune system. Inflammation influences CKD development either directly, due to acute kidney injury (AKI), and post-renal acute kidney injury, or indirectly by fostering atherosclerosis leading to CKD and the subsequent kidney function deterioration $(10,11,12)$. The extent of inflammatory response is associated with the severity of CKD and linearly increases with the progression of renal failure (8).

Activated blood macrophages produce cytokines as essential mediators to help determining the extent of immune response which is extremely important in order to mediate the progression of the disease (11). The pathophysiological balance between pro- and anti-inflammatory cytokines is necessary to determine the net effect of an inflammatory response (2). These cytokines are both activated in acute- and chronic kidney diseases $(2,11,12$, 13). The most known pro-inflammatory cytokines include; interleukin-1 (IL-1), interleukin-2 (IL- 2), interleukin-6 (IL-6), interleukin-8 (IL-8), interleukin-17 (IL-17) and tumor necrosis factor- $\alpha$ (TNF- $\alpha)(14,15)$. Risk factors for chronic inflammation include age progression, obesity, type 2 diabetes, and chronic recurrent infections $(2,5,6,13)$.

The development of CKD is accompanied by an increase in serum creatinine levels (16) and other CKD related markers including serum urea, urine albumin and to lesser extent serum uric acid (10, 17, 18, 19, 20). Nevertheless, serum creatinine is measured routinely in order to diagnose individuals suspected with AKI (16, 21). It is also used to evaluate the improvement and /or deterioration in kidney function throughout AKI and CKD treatment $(16,18)$. Although CKD is mediated by inflammation at different stages of disease development $(2,11$, 13), however evidence for their direct association with disease risk remains inconclusive. The current study was designed to evaluate the relationship between interleukins
IL-2 and IL-17 concentration and kidney function markers in men with CKD admitted to the largest public hospital in the city of Ramadi, the capital of Al-anbar province, Iraq.

\section{Materials and Methods Ethical Clearance}

One hundred and fifteen Iraqi-Arabs men is the actual number of the participants in the current study. The study protocol was approved ethically by the University of Anbar (No:166SC-2021), and every participant has signed a written consent form after listening to a brief presentation about the project.

\section{Case definition and CKD confirmation}

In the current study, a confirmed CKD case was defined as; any individual with an estimated glomerular filtration rate (eGFR) of less than $60 \mathrm{~mL} / \mathrm{min} / 1.73 \mathrm{~m}^{2}$ body surface area or an evidence of kidney damage (even when eGFR is normal), detected by abnormal increase of serum creatinine and urine albumin, persisting for more than 3 months as established previously (18).

\section{Collection of the study participants}

This study was conducted during the time from April 2021 to August 2021. All patients were evaluated before the treatment was initiated. To make the process of collecting controls much easier, we have asked each patient with CKD to bring a relative, or a friend. We further managed to recruit more controls from the same hospital from where patients were recruited.

\section{Clinical examination}

Scheduled comprehensive interviews were conducted at the hospital by highly trained nurses in order to collect demographic, socioeconomic and anthropometric details. Details on individuals' medical history were also obtained. Blood pressure was obtained using a fully automated machine (Omran blood pressure monitor). All participants of the current study went through a clinical examination to ensure that each participant (patients and controls) fits the study criteria. 


\section{Inclusion and exclusion criteria}

Iraqi men aged 30 to 70 years old, living in the province of Al-anbar and who have been willing to join the study were included in the current study. In order to avoid any possible interlude of genetics of other races, we included Arabs only.

To eliminate the effect of any pathophysiological and pathogenetic factors that might influence the results of the study, subjects were excluded if they were not Arab or had diabetes mellitus or abnormal blood values. Based on medical records history (supported by self-confirmation report), individuals with inflammatory diseases and/or infectious diseases such as rheumatoid arthritis, psoriasis, hyperthyroidism, inflammatory bowel disease (IBD), human immune deficiency virus (HIV), hepatitis B virus (HBV), hepatitis $\mathrm{C}$ virus (HCV), covid19, influenza, lung infection and tonsillitis were also excluded. Further subjects were excluded if they were having known muscle disease, or performed vigorous exercise training within 3 months or had a job that required heavy lifting. All participants of the current study were requested to give-up smoking and alcohol consumption.

\section{Collection and handling of blood samples}

One week before blood withdrawal subjects abstained from analgesic use and physical activity.

All participants were asked not to drink or eat anything after $9 \mathrm{pm}$ the night before their blood were taken. After 8 to 10 hours overnight fast, $10 \mathrm{ml}$ of fresh blood was drawn from the antecubital vein. In order to comfort the participants of the current study, well trained nurses explained the procedure to each participant before puncture of the vein using disposal plastic syringe. Two types of tubes were used for blood collection (yellow and purple). All blood samples were collected between 7.30 to $8.30 \mathrm{am}$, blood samples then were kept for 30 minutes in a cold room before samples of the yellow tubes were separated from cells by centrifugation at $3000 \mathrm{x} g$ for 10 minutes. The plasma was snap frozen and stored at $-20{ }^{\circ} \mathrm{C}$ until testing. Purple tubes were left on a blood laboratory mixer, before processing to get glycohaemoglobin A1C (HbA1c) measurements that have been reported as a percentage of total hemoglobin. All samples were processed similarly according to standardized protocol.

\section{Collection and handling of urine samples}

Urine samples were collected in sterile containers early in the morning before the withdrawal of blood samples. Then later on the same day, a sufficient sample of urine was transferred slowly to a $6 \mathrm{ml}$ sterile plain tube using a $50 \mathrm{ml}$ disposal plastic syringe after the needle was removed. All samples were stored in a fridge until enough samples were collected and analyzed in triplicate. All participants were requested to get midstream uninterrupted urine sample. An illustrative short cartoon movie has been displayed by one of the project's staff who was designated to describe a step-by-step midstream urine collection in order to help all participants to apply the exact instructions to collect a representative, clean midstream urine sample.

\section{Diagnosis of diabetes}

Participants were excluded if their average fasting glucose levels were more than (125 $\mathrm{mg} / \mathrm{L}$ ) and non-fasting of $\geq 200 \mathrm{mg} / \mathrm{L}$ and their glycohaemoglobin A1C (HbA1c) was more than 7 . In addition, we have also relied earlier on individual medical records and treatment history to exclude inapplicable volunteers.

\section{Measuring of serum plasma interleukins}

Plasma levels of IL-2, IL-17 have been measured using an Enzyme Linked Immunosorbent Assay kit (ELISA). ELISA kits were provided by My-bio-source company, USA) and kidney function tests such as blood urea, serum creatinine, serum uric acid and urine albumin were also performed in all subjects using an autoanalyzer. Each sample was measured in triplicate and the mean value of each sample was then considered ready for statistical analysis. Microalbuminurea was defined as the 
excretion of 30-150 $\mathrm{mg}$ of protein per day as a sign of early renal disease described previously (22). The protocol by the assay manufacturer was strictly followed and all samples were tested undiluted.

\section{Statistical analysis}

Statistical analysis was performed using GraphPad prism version 7.04 and Software Excel 2016. Descriptive statistics for each parameter consisted of the standard error (SE), the mean and the standard deviation (SD). The level of statistical significance was set as long as $P$ value is less than 0.05 , the relationship between IL-2, IL-17 and other parameters of CKD patients and controls were evaluated using Pearson's correlation $(\mathrm{r}=-1$ to 1$)$. Logistic regression model was performed to justify the relationship between the interleukins and CKD variables, and to eliminate any possible interference of other variables. Variables that may confound the results of the current study are; Age, BMI, waist-circumference and waist-hip-ratio.

\section{Results}

Seventy-eight patients and seventy-five controls have signed consent forms to be enrolled in the current study. After the application of inclusion and exclusion criteria and based on medical history of each participant, twenty-seven patients with CKD were excluded as five have been diagnosed previously with $\mathrm{HBV}$ and the rest were diabetic. Furthermore, six more patients were excluded after their blood values (fasting glucose and HbA1C) showed they have type 2 diabetes. Out of the total number of the individuals who had joined the control group only five were excluded as they were diagnosed with type 2 diabetes. Thus, the final number who satisfied the inclusion criteria of the current study were forty-five men with prevalent CKD (age range 30-70 years, mean age; 53.66 \pm 6.03 , mean BMI's: 28.91 \pm 3.39 ; mean waist circumference: $106.41 \pm 8.81$; mean SBP: $140.39 \pm 15.90$; mean DBP: 86.77 \pm 10.20 ; mean FSG: $118 \pm 4.71$; mean HbA1c: $5.67 \pm 1.38$ ) and seventy healthy men at same age range (mean age: $51.64 \pm 5.59$; mean BMI: $26.83 \pm 1.85$; mean waist circumferences: 93.25 \pm 6.46 ; mean SBP: $123.34 \pm 5.91$; mean DBP: $80.95 \pm 4.39$; mean FSG: $101 \pm 5.24$; mean HbA1c: $5.1 \pm 0.97$ ) with no known active infectious and other inflammatory diseases served as controls.

\section{CKD parameters}

Compared with controls, we have observed a significant increase in serum creatinine $(\mathrm{p}<$ $0.001)$, serum urea $(\mathrm{p}<0.001)$, and urine albumin $(\mathrm{p}<0.001)$ as seen in figures 1,2 , and 3. No such relationship was seen in serum uric acid concentrations between CKD patients and their controls (Fig. 4).

\section{The relationship between interleukins and CKD parameters}

The current study has shown for a first time a significant liner relationship between creatinine level and IL-2 ( $p<0.001)$ and IL-17 $(p<0.001)$ as shown in Figure 1, respectively. These interleukins have also associated with significant increase in serum urea $(\mathrm{p}<0.001)$, and urine albumin ( $\mathrm{p}<0.001)$, respectively (Figs. 2 and 3). No significant differences in serum uric acid were observed between patients with CKD and controls, neither there were any association with IL-2 and IL-17 levels (Fig. 4).

\section{Discussion}

In clinical and pathology reports, serum creatinine concentrations are commonly used to diagnose patients with AKI and to get a general evaluation for treated individuals $(16,17,21)$. In addition, serum creatinine together with urine albumin has been used to monitor patients with CKD throughout the treatment and to evaluate any improvement or deterioration in kidney function $(16,18,22,23)$. In patients with CKD, serum creatinine concentrations steadily increase, together with serum urea and urine albumin in parallel with the deterioration of kidney function $(17,20,22)$. Although limited numbers of previous studies have shown a significant increase in serum uric acid level in patients with $\mathrm{AKI}$ and $\mathrm{CKD}$, it is not frequently 
ordered in daily clinical practice (24). Deterioration of kidney function in patients with CKD occurs gradually, it is irreversible process and is usually associated with significantly high levels of inflammatory markers. Chronic inflammation is an established risk factor for CKD and has been linked to marked increase in morbidity and mortality rate $(2,19,25)$. In the current study, we have observed a significant increase in serum creatinine $(p<0.001)$, serum urea $(p<0$. $001)$ and urine albumin $(p<0.001)$ in patients with CKD compared with controls. In contrast, no significant relationship was observed in serum uric acid between CKD patients and their controls.

The elevation of serum creatinine concentration was associated with a significant increase in both IL-2 ( $\mathrm{p}<0.001)$ and IL-17 ( $\mathrm{p}<$ 0. 001) levels (Fig. 1). Similarly, IL-2 and IL17 levels were strongly associated with urine albumin concentrations (Fig. 2), and serum urea (Fig.3), suggesting that these interleukins may play critical roles in the pathogenesis of CKD. No correlation was detected between serum uric acid and IL-2 and IL-17 levels (Fig.4). To our knowledge, this is first study performed to evaluate the relationship between these interleukins and the classic biochemical markers of CKD. The prognostic importance of elevated IL-2 and IL17 levels are independent of and additive to the prognostic effect of creatinine and other classic kidney function markers including serum urea and urine albumin. A previous study targeted interleukins in relationship to lupus nephritis histopathology and therapy response has suggested that high levels of IL-17 could be related to therapy resistance, severity of the disease or disease phenotype (26).The high levels of interleukins have been reported in wide range of diseases such as metabolic syndrome (27) and different types of cancer (28).
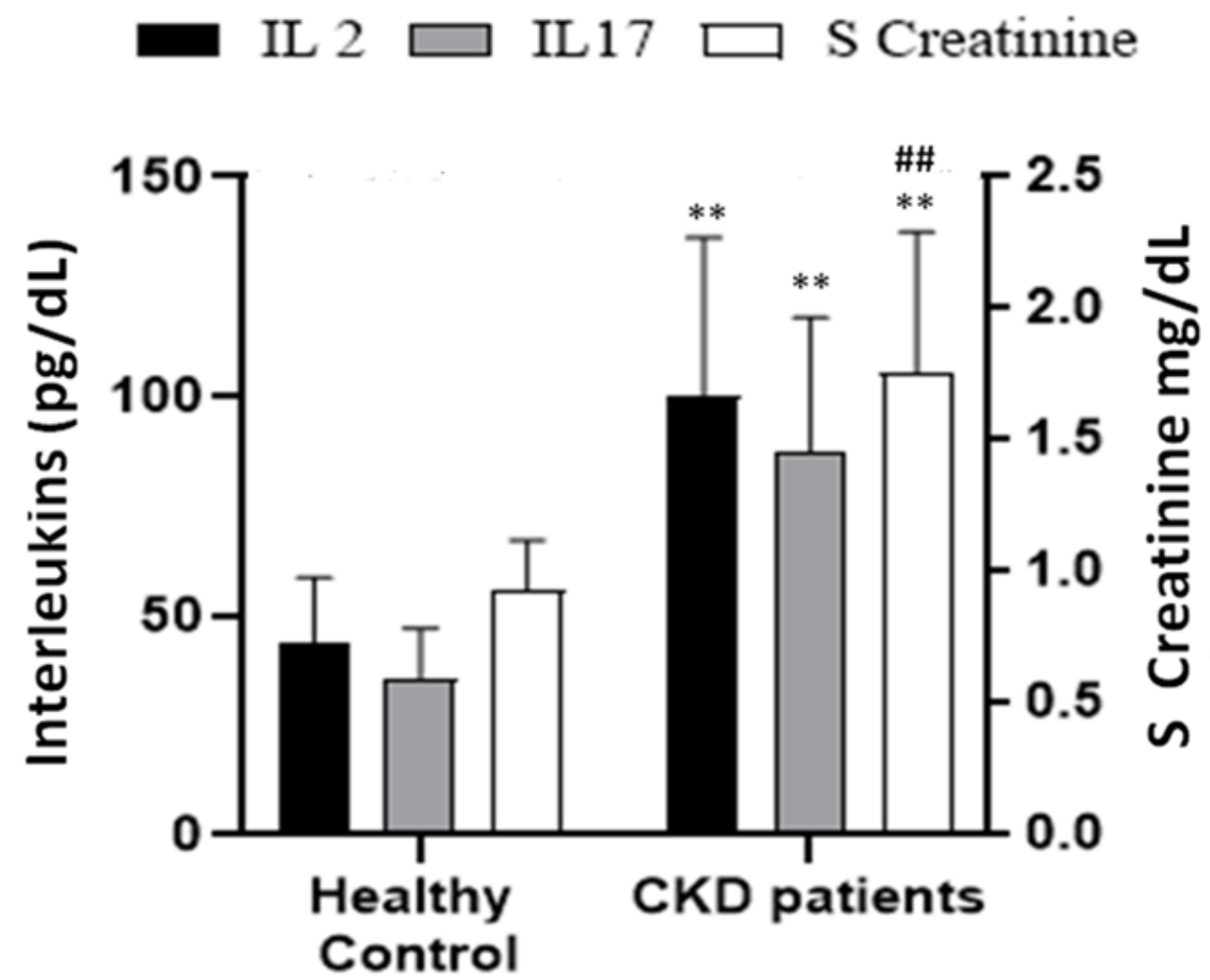

Fig. 1. The relationship between serum IL-2, IL17 and serum creatinine concentrations in patients with CKD and their controls. Compared with controls, a significant increase in serum IL-2 and IL-17 levels ( $\left.{ }^{* * *} \mathrm{p}<0.001\right)$ were correlated with a significant increase in serum creatinine concentrations $\left({ }^{* *} \mathrm{p}<0.001\right)$. Significant differences in serum creatinine concentrations have been detected between cases and controls $\left({ }^{\#} \mathrm{p}<0.001\right)$. 




Fig. 2. The relationship between serum IL-2, IL-17 and urine albumin concentrations in patients with CKD and their controls. Compared with controls, a significant increase in serum IL-2 and IL-17 levels $\left({ }^{* *} \mathrm{P}<0.001\right)$ were correlated with a significant increase in urine albumin concentrations $\left({ }^{* *} \mathrm{p}<0.001\right)$. Significant differences in urine albumin concentrations have been detected between cases and controls $\left({ }^{\# \#} \mathrm{p}<0.001\right)$.
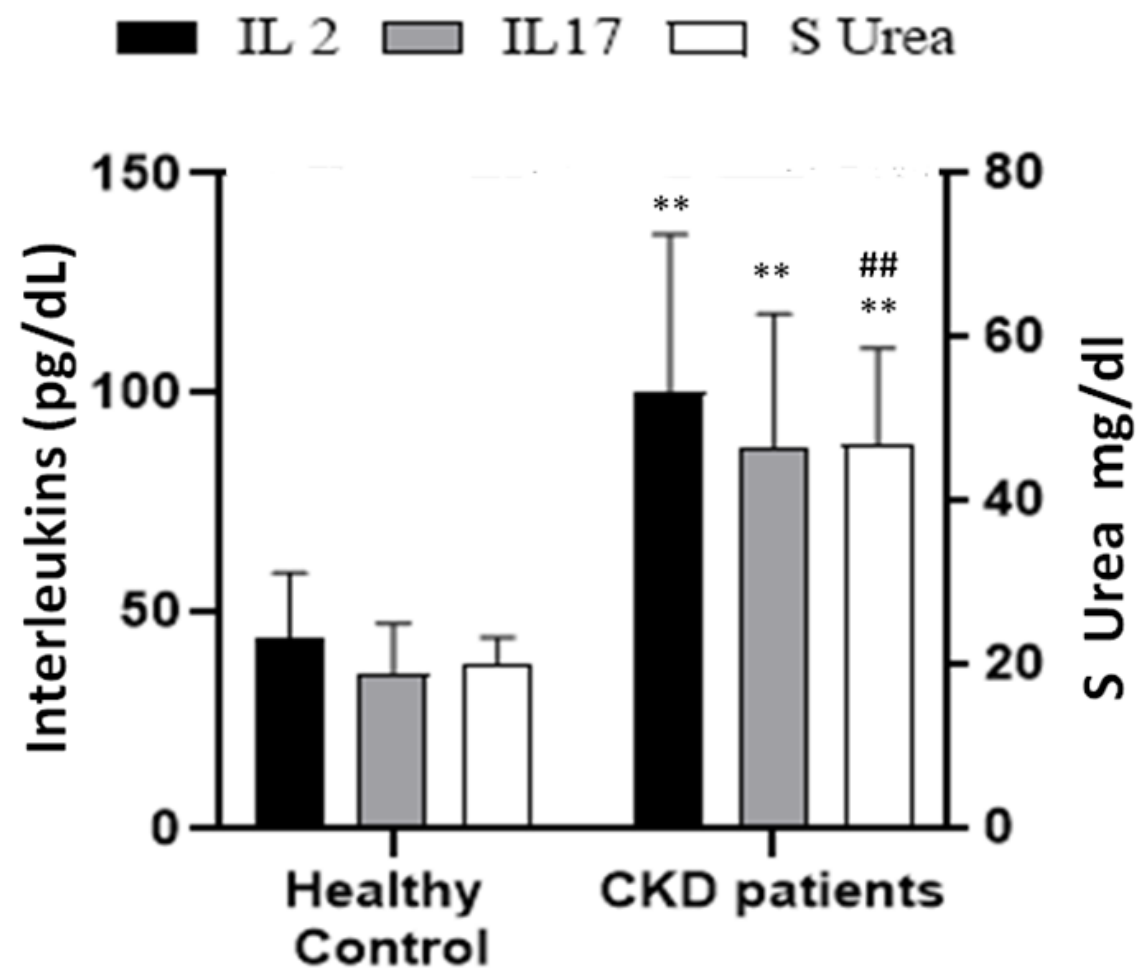

Fig. 3. The relationship between serum IL-2, IL-17 and serum urea concentrations in patients with CKD and their controls. Compared with controls, a significant increase in serum IL-2 and IL-17 levels $\left.{ }^{* *} \mathrm{p}<0.001\right)$ were correlated with a significant increase in serum urea concentrations $\left({ }^{* *} \mathrm{p}<0.001\right)$. Significant differences in serum urea concentrations have been detected between cases and controls $\left({ }^{\#} \mathrm{p}<0.001\right)$. 



Fig. 4. The relationship between serum IL-2, IL-17 and serum uric acid concentrations in patients with CKD and their controls. Compared with controls, a significant increase in serum IL-2 and IL-17 levels ( $\left.{ }^{* *} \mathrm{p}<0.001\right)$ were not correlated with a significant increase in serum uric acid concentrations. No significant differences in serum uric acid concentrations have been detected between cases and controls $\left({ }^{* *} \mathrm{p}<0.001\right)$.

Compared with individuals in the control group, patients with CKD especially those at advance stages of the disease (i.e. stages 3, 4 and 5) have significantly higher levels of inflammatory cytokines such as tumor necrosis factor- $\alpha$ (TNF- $\alpha$ ) and interleukin-6 (IL-6) (29). A simple explanation of the former is because a decrease in glomerular filtration rate (GFR) usually induces a significant decrease in the clearance of these cytokines. In addition, and because the body continues to secrete these cytokines, their circulating levels continue escalating $(25,30)$. Similarly, elevated serum IL-2 and serum IL-17 levels in the current study most probably was due to previously mentioned reasons (30). The relationship between creatinine concentration and IL-2 and IL-17 has been inter-correlated positively both with BMI $(\mathrm{p}<0.01)$ and waist-circumferences $(p<0.001)$, and such relationship was robust in obese and very obese patients $(\mathrm{p}<0.001)$. Furthermore, we have observed a significant increase in serum creatinine concentrations in parallel with age progression. Thus, when CKD patients were divided into two groups based on their age, older CKD patients $(>50$ years) found to have higher concentrations of creatinine compared with younger CKD patients ( $<50$ years). Similarly, urine albumin or albuminurea and serum urea concentrations were both significantly higher among older patients compared with younger patients, which is in parallel with previous literature (5, 6). Among patients with CKD, older age is associated with poor prognosis and high morbidity and mortality rate $(31,32)$. Deterioration in kidney function among older patients with CKD could be due to a longer period living with the disease and associated health risks. The high prevalence of arthrosclerosis and cardiovascular disease (CVD) related co-morbidities including hypertension in older patients with CKD likely contributes to a significant decrease in life expectancy compared with younger CKD patients $(31,33)$. In contrast, the vast majority of healthy elderly, the decline in kidney function is modest and cannot be predicted (33, 34). 
A previous study reported an inverse relationship between serum albumin and the risk of declining kidney function which corresponds with our results (20). However, in the current study and in order to evaluate kidney function, we have measured urine albumin rather than serum albumin. Urine albumin or albuminurea in addition to serum creatinine is usually measured to evaluate kidney function in individuals suspected with kidney injury and newly diagnosed patients with diabetes $(17,18)$. Furthermore, we have not calculated albumin creatinine ratio (ACR), because using ACR provide no advantages compared with measuring urine albumin alone (35). Compared with ACR, measuring urine albumin is more convenient in daily laboratory medicine practice for both patients and medical staff as previously reported (35). In patients newly diagnosed with diabetes (with or without CKD), a urine albumin concentration test is frequently requested by clinicians to have a clearer image on serum glucose spike, hyperglycaemia and to get an impression of possible kidney injury due to uncontrolled diabetes $(17,22)$. The urine albumin test is also requested when monitoring patients with $\mathrm{AKI} / \mathrm{CKD}$ under treatment. In laboratory medicine, urine albumin is usually assessed either by using an automated approach or by using a urine dipstick, while light microscopy is commonly used for further urine studies when required. Urine dipstick testing is an easy, cheap and effective screening approach to detect CKD in primary care facility. It instantly gives evaluation for protein and glucose levels and all these features enable healthcare practitioner to request the test any time of the day $(17,22,35)$. Furthermore, urine dipstick testing can be used in urban as well as in remote societies without the need for an established biochemistry laboratory $(35,36)$. Japan has considered urinalysis using dipstick tool compulsory even for school children, and Japanese nationwide screening program for AKI/CKD using urine albumin and serum creatinine was associated with a significant decline in the incidence/prevalence of $\mathrm{CKD}$ and related morbidity and mortality rate $(6,36,37)$.

Depending on the severity of CKD, serum urea concentration in patients with kidney dysfunction can fluctuate from mildly to severely elevated. In severe CKD cases serum urea usually exceeds $50 \mathrm{mmol} / \mathrm{L}(17,18,38)$. Although we had patients in the current study that belong to developed stages of the disease (stage 3 and beyond), but because the majority of the patients are still at earlier CKD stages (stage 1 and 2), we determined a mean value for serum urea of $<50 \mathrm{mmol} / \mathrm{L}$. The deterioration of kidney function in patients with CKD is associated with a gradual decrease in urinary excretion of urea, resulting in elevated urea concentrations in the blood. Earlier studies reported an inverse association between plasma urea concentration and eGFR value, suggesting that the serum urea test might be important in the evaluation of kidney function deterioration, irrespective of the cause (39). However, a serum urea test has low sensitivity and low specificity and can be affected by high protein diet and individual's hydration status. As a result, slight to moderate elevation in serum urea does not necessarily indicate kidney dysfunction, while normal urea concentration does not rule out kidney disease either (38). Clinically, the kidneys of an individual with CKD usually lose $50 \%$ of their function (calculated as $50 \%$ reduction in GFR) before serum urea crosses the upper normal range $(17,18,39)$. Due to its prognostic efficacy, a plasma creatinine test continues to be the first line test when evaluating kidney function. Furthermore, a serum creatinine test is also preferred when monitoring and treating patients with $\mathrm{AKI}$ and CKD $(17,18,23)$.

Previously, it has been suggested that interleukins in general may have pro- and antiinflammatory response as their level increased at earlier stages of CKD development and might steadily decrease at later stages (40). Unfortunately, due to limited sample size and the threatening Covid-19 epidemic (41) which is associated with subsequent shutdown of 
most private and public sectors in the country, we were not able to perform follow-up studies. The strong association between CKD's markers and IL-2 and IL-17 levels suggests that these interleukins could be used with CKD markers to evaluate kidney function in health and disease and thus, it may help in monitoring patients with CKD under treatment. Expectedly, we have observed significantly higher levels of interleukins $(p<0.001)$ among older CKD patients ( $>50$ year) compared with younger patients $(<50$ years). Previous studies have reported a significant increase in inflammatory cytokines with age progression $(11,12,13,25)$, which is in support of the current data. Older patients with CKD have spent a longer period battling with the disease, and most of them are at developed stages (stage 3 and beyond) compared with younger patients $(<50$ years old $)$. Furthermore, age progression is associated with a significant decline in health in parallel with an increased risk of metabolic diseases such as obesity, hypertension and CVD $(4,9,10,11)$.

The novelty of the current study involves the evaluation of IL-2 and IL-17 levels in relation to creatinine and other CKD markers. Furthermore, we have followed strict exclusion/inclusion criteria that enabled us to exclude all participants with inflammatory related diseases in order not to confound the results and to be able to justify our conclusion. Our study however, is limited due to a

\section{References}

1. Jager KJ, Kovesdy C, Langham R, Rosenberg $\mathrm{M}$, Jha V, Zoccali C. A single number for advocacy and communication-worldwide more than 850 million individuals have kidney diseases. Kidney Int. 2019;95(5):1048-1050.

2. Akchurin OM, Kaskel F. Update on inflammation in chronic kidney disease. Blood Purif. 2015;39(1-3):84-92.

3. Foreman KJ, Marquez N, Dolgert A, Fukutaki K, Fullman N, McGaughey M, et al. Forecasting life expectancy, years of life lost, and all-cause and cause-specific mortality for 250 causes of death: reference and alternative scenarios for relatively small sample size, which probably was a direct result of the current pandemic. The looming situation due to covid-19 pandemic has a severe impact on the participation process of the current study and this is probably the situation everywhere else. However, it has become clear that CKD is a risk factor for severe COVID-19 (41).

Compared with healthy controls, serum IL2 and IL-17 levels were increased significantly in patients with CKD. Increased IL-2 and IL17 levels in patients with CKD were in parallel with a significant increase of creatinine, urine albumin and serum urea concentration. These results suggest an important role of IL-2 and IL-17 in the pathogenesis of CKD. Nevertheless, a large prospective cohort studies are necessary to establish a normal reference range for these interleukins and to confirm their diagnostic efficacy as additional markers in patients with CKD.

\section{Acknowledgements}

We would like to thank clinicians, nurses and laboratory technologists in Ramadi general hospital for their assistance in this study. We also thank the Faculty of Sciences, University of Anbar, Iraq for their technical support. Finally, we would like to express our gratitude to all the participants of this study.

The authors declare that they have no conflicts of interest.

2016-40 for 195 countries and territories. Lancet 2018;392(10159):2052-2090.

4. Ricardo AC, Anderson CA, Yang W, Zhang X, Fischer MJ, Dember LM, et al. Healthy lifestyle and risk of kidney disease progression, atherosclerotic events, and death in CKD: finding from the Chronic Renal Insufficiency Cohort (CRIC) Study. Am J Kidney Dis. 2015;65(3):412-24.

5. Bleyer AJ, Shemanski LR, Burke GL, Hansen KJ, Appel RG. Tobacco, hypertension, and vascular disease: Risk factors for renal functional decline in an older population. Kidney Int. 2000;57(5):2072-9. 
6. Yamagata K, Ishida K, Sairenchi T, Takahashi $\mathrm{H}$, Ohba S, Shiigai T, et al. Risk factors for chronic kidney disease in a community-based population: a 10-year follow-up study. Kidney Int. 2007;71(2): 159-66.

7. Collaborators GRFS. Global regional and national comparative risk assisment of 76 behavioral environment, occupational and metabolic risk or clusters of risks in 188 countries 1990-2013: a systemic analysis for the GBD 2013. Lancet. 2015;386(10010):2287-323.

8. Kim MG, Kim SC, Ko YS, Hee YL, Jo SK, Cho W. The role of M2 macrophages in the progression of chronic kidney disease following acute kidney injury. PloS One 2015;10(12):e0143961.

9. Kon V, Linton MRF, Fazio S. Atherosclerosis in chronic kidney disease: the role of macrophages. Nat Rev Nephrol. 2011;7(1):45-54. 10. Levey AS, Beto JA, Coronado BE, Eknoyan G, Foley RN, Kasiske BL, et al. Controlling the epidemic of cardiovascular disease in chronic renal disease: what do we know? What do we need to learn? Where do we go from here? National Kidney Foundation Task Force on Cardiovascular Disease. Am J Kidney Dis. 1998;32:853-906.

11. Carrero JJ, Park SH, Axelsson J, Lindholm B, Stenvinkel P. Cytokines, atherogenesis, and hypercatabolism in chronic kidney disease: a dreadful triad. Semin Dial. 2009;22(4):381-6.

12. Silverstein DM. Inflammation in chronic kidney disease: role in the progression of renal and cardiovascular disease. Pediatr Nephrol. 2009;24(8):1445-52.

13. Koelman L, Pivovarova-Ramich O, Pfeiffer AFH, Grune T, Aleksandrova K. Cytokines for evaluation of chronic inflammatory status in ageing research: reliability and phenotypic characterization. Immunity and Ageing. 2019;16:11.

14. Mittal RD, Manchanda PK. Association of interleukin (IL)-4 intron-3 and IL-6 -174 G/C gene polymorphism with susceptibility to endstage renal disease. Immunogenetics. 2007;59(2):159-65.

15. Fasoulakis Z, Kolios G, Papamanolis V, Kontomanolis EN. Interleukins associated with breast cancer. Cureus. 2018;10(11):e3549.
16. Kellum JA, Sileanu FE, Bihorac A, Hoste EA, Chawla LS. Recovery after acute kidney injury. Am J Respir Crit Care Med. 2017;195(6); 784-791.

17. Traynor J, Mactier R, Geddes CC, Fox JG. How to measure renal function in clinical practice. BMJ. 2006;333(7571):733-737.

18. Nickolas TL, Barasch J, Devarajan P. Biomarkers in acute and chronic kidney disease. Curr Opin Nephrol Hypertens. 2008;17(2):12732.

19. Ortiz A, Covic A, Fliser D, Fouque D, Goldsmith D, Kanbay M, et al. Epidemiology, contributors to, and clinical trials of mortality risk in chronic kidney failure. Lancet. 2014;383(9931):1831-43.

20. Lang J, Katz R, IX JH, Gutierrez OM, Peralta $\mathrm{CA}$, Parikh CR, et al. Association of serum kidney albumin levels with kidney function decline and incident chronic kidney disease in elders. Nephrol Dial Transplant. 2018;33(6):986-992.

21. Forni LG, Darmon M, Ostermann M, Straaten HM O-V, Pettilä V, Prowle JR, et al. Renal recovery after acute kidney injury. Intensive Care Med. 2017;43(6):855-866.

22. Simerville JA, Maxted WC, Pahira JJ. Urinalysis: A comprehensive review. Am Fam Physician. 2005;71(6):1153-1162.

23. Levin A, Stevens PE, Bilous RW, Coresh J, De Francisco ALM, De Jong PE, et al. Kidney Disease Improving Global Outcomes (KDIGO) CKD Work Group-KDIGO 2012 clinical practice guidelines for the evaluation and management of chronic kidney disease. Kidney Int. 2013;3(1):1-150.

24. Johnson RJ, Nakagawa T, Jalal D, SánchezLozada LG, Kang DH, Ritz E. Uric acid and chronic kidney disease: which is chasing which?. Nephrol Dial Transplant. 2013;28(9):2221-2228. 25. Stenvinkel $P$, Alvestrand A. Inflammation in end-stage renal disease: sources, consequences and therapy. Semin Dial. 2002;15(5):329-37.

26. Zickert A, Amoudruz P, Sundström Y, Rönnelid J, Malmström V, Gunnarsson I. IL-17 and IL-23 in lupus nephritis - association to histopathology and response to treatment. BMC Immunol. 2015;16(1):7.

27. Mohammadi M, Gozashti MH, Aghadavood M, Mehdizadeh MR, Hayatbakhsh MM. Clinical 
significance of serum TNF-a and IL-6 levels in patients with metabolic syndrome. Rep Biochem Mol Biol. 2017;6(1):74-79.

28. Raghunathachar Sahana K, Akila P, Prashant V, Sharath Chandra B, Nataraj SM. Quantitation of vascular endothelial growth factor and interleukin- 6 in different stages of breast cancer. Rep Biochem Mol Biol. 2017;6(1):33-39.

29. Lee BT, Ahmed FA, Lee Ham L, Teran FJ, Chen C-S, Liu Y, et al. Association of C-reactive protein, tumor necrosis factor-alpha, and interleukin-6 with chronic kidney disease. BMC Nephrol. 2015;16:77.

30. Stenvinkel P, Ketteler M, Johnson RJ, Lindholm B, Pecoits-Filho R, Riella M, et al. Interleukin-10, IL-6 and TNF-alpha: central factors in the altered cytokine network of endstage renal disease - the good, the bad and the ugly. Kidney Int. 2005;67(4):1216-1233.

31. Tamura MK. Incidence, management, and outcome of end-stage renal disease in the elderly. Curr Opin Nephrol Hypertens. 2009;18(3):252-257. 32. Hallan S I, Matsushita K, Sang Y, Mahmoodi BK, Black C, Ishani A, et al. Age and the association of kidney measures with mortality and end-stage renal disease. JAMA. 2012;308(22):2349-60.

33. Nitta K, Okada K, Yanai M, Takahashi S. Aging and chronic kidney disease. Kidney Blood Press Res. 2013;38(1):109-20.

34. De Nicola L, Minutolo R, Chiodini P, Borrelli S, Zoccali C, Postorino M, et al. The effect of increasing age on the prognosis of non- dialysis patients with chronic kidney disease receiving stable nephrology care. Kidney Int. 2012;82(4):482-488.

35. Derhaschnig U, Kittler H, Woisetschlager C, Bur A, Herkner H, Hirschl MM. Microalbumin measurement alone or calculation of the albumin creatinine ratio for the screening of hypertension patients?. Nephrol Dial Transplant. 2002;17(1):8185.

36. Pugia MJ, Murakami M, Lott JA, Ohta Y, Kitagawa T, Yamauchi K, et al. Screening for proteinuria in Japanese schoolchildren: a new approach. Clin Chem Lab Med. 2000;38(10):975-82.

37. Imai E, Yamagata K, Iseki K, Iso H, Horio M, Mkino $\mathrm{H}$, et al. Kidney disease screening program in Japan: history, outcome and perspectives. Clin J Am Soc Nephrol. 2007;2(6):1360-6.

38. Salazar JH. Overview of urea and creatinine. Lab Medicine 2014; 45(1): e19-e20.

39. Lyman JL. Blood urea nitrogen and creatinine. Emerg Med Clin North Am. 1986;4(2):223-233.

40. Scheller J, Chalaris A, Schmidt-Arras D, RoseJohn S. The pro-and anti-inflammatory properties of the cytokine interleukin-6. Biochim Biophys Acta. 2011;1813(5):878-88.

41. ERA-EDTA Council; ERACODA Working Group. Chronic kidney disease is a key risk factor for severe COVID-19: a call to action by the ERA-EDTA. Nephrol Dial Transplant. 2021;36(1):87-94. 